\title{
Larvicidal and Pupicidal Activities of Alizarin Isolated from Roots of Rubia cordifolia Against Culex quinquefasciatus Say and Aedes aegypti (L.) (Diptera: Culicidae)
}

\author{
MR Gandhi ${ }^{1}$, AD Reegan ${ }^{1}$, P Ganesan $^{1}$, K Sivasankaran ${ }^{1}$, MG Paulraj $^{1}$, K Balakrishna ${ }^{1}$, \\ S IGNACIMUTHu, ${ }^{1,2}$, NA AL - DHABI ${ }^{3}$ \\ 'Division of Vector Control, Entomology Research Institute, Loyola College, Chennai Tamil Nadu, India \\ ${ }^{2}$ Visiting Professor Programme, Deanship of Research, King Saud Univ, Riyadh, Saudi Arabia \\ ${ }^{3}$ Dept of Botany and Microbiology, Addiriyah chair for Environmental Studies, College of Science, King Saud Univ, Riyadh, Saudi Arabia
}

\section{Keywords}

Bioassay, chikungunya, dengue, lymphatic filariasis, vector control

\section{Correspondence \\ S Ignacimuthu, Entomology Research Institute, Loyola College, Nungambakkam, Chennai 600 034, Tamil Nadu, India; eriloyola@hotmail.com}

Edited by Raul NC Guedes - UFV

M R Gandhi and A D Reegan contributed equally to this work.

Received 14 October 2015 and accepted 23 February 2016

Published online: 23 March 2016

(C) Sociedade Entomológica do Brasil 2016

\begin{abstract}
The mosquitocidal activities of different fractions and a compound alizarin from the methanol extract of Rubia cordifolia roots were evaluated on larvae and pupae of Culex quinquefasciatus Say and Aedes aegypti (L.) (Diptera: Culicidae). Larvae and pupae were exposed to concentrations of 2.5, 5.0, 7.5 and $10 \mathrm{ppm}$ for fractions and 0.5, 1.0, 1.5 and $2.0 \mathrm{ppm}$ for compound. After $24 \mathrm{~h}$, the mortality was assessed and the $\mathrm{LC}_{50}$ and $\mathrm{LC}_{90}$ values were estimated for larvae and pupae. Among the 23 fractions screened, fraction 2 from the methanol extract of $R$. cordifolia showed good mosquitocidal activity against $C$. quinquefasciatus and $A$. aegypti. $\mathrm{LC}_{50}$ and $\mathrm{LC}_{90}$ values of fraction 2 were 3.53 and $7.26 \mathrm{ppm}$ for C. quinquefasciatus and 3.86 and 8.28 ppm for A. aegypti larvae, and 3.76 and $7.50 \mathrm{ppm}$ for $C$. quinquefasciatus and 3.92 and $8.05 \mathrm{ppm}$ for A. aegypti pupae, respectively. Further, the isolated compound alizarin presented good larvicidal and pupicidal activities. $\mathrm{LC}_{50}$ and $\mathrm{LC}_{90}$ values of alizarin for larvae were 0.81 and $3.86 \mathrm{ppm}$ against $C$. quinquefasciatus and 1.31 and $6.04 \mathrm{ppm}$ for $A$. aegypti larvae, respectively. Similarly, the $\mathrm{LC}_{50}$ and $\mathrm{LC}_{90}$ values of alizarin for pupae were 1.97 and $4.79 \mathrm{ppm}$ for C. quinquefasciatus and 2.05 and $5.59 \mathrm{ppm}$ for $A$. aegypti pupae, respectively. The structure of the isolated compound was identified on the basis of spectroscopic analysis and compared with reported spectral data. The results indicated that alizarin could be used as a potential larvicide and pupicide.
\end{abstract}

\section{Introduction}

Mosquitoes are small insects in the order Diptera. Many species of mosquitoes serve as important vectors of several diseases. Culex quinquefasciatus Say is an important vector of lymphatic filariasis in tropical and subtropical regions, as it vectors Wuchereria bancrofti (Holder 1999). According to World Health Organization report (1984), about 90 million people worldwide are infected with $W$. bancrofti and ten times more people are at the risk of being infected. Alone in India, 25 million people harbour microfilaria (mf) and 19 million people suffer from filarial disease manifestations (NICD 1990, Reegan et al 2015). Aedes aegypti (L.) is the primary vector involved in the transmission of dengue, chikungunya and Zika viruses (Harrington et al 2005, Kannathasan et al 2011, Yakob \& Walker 2016). Many Asian countries including India are endemic for dengue fever. Many sporadic dengue cases have been reported from various parts of India (Akram \& Ahmed 2005) and a major outbreak was recorded during 2012. During this outbreak, a total 
of 5376 dengue cases and 39 deaths were detected in the state Tamil Nadu, southern India (Kannan 2012, Reegan et al 2014). The climatological condition also favoured rapid increase of $A$. aegypti populations.

For the past several decades, synthetic insecticides have been used against the aquatic stages of vector mosquitoes. The usage of synthetic insecticides-pyrethroids, organophosphates, organochlorines and carbamates-is increasing year after year, and continuous application of these insecticides pose a major threat to environment and human health (Shaalan et al 2005, Sutthanont et al 2010, Madhu et al 2010, Bayen 2012). Phytocompounds isolated from plants are target specific and safe to all associated organisms. Hence, plant-derived products would be a good alternative to synthetic insecticides.

Decoction from $R$. cordifolia roots is prescribed to cure jaundice, paralytic affections and urinary dysfunctions (Devi Priya \& Siril 2014). Roots of $R$. cordifolia have also been used as astringent, thermogenic, febrifuge, antidysenteric, antihelmintic, galactopurifier, ophthalmic and rejuvenant and used to treat cough, bladder and kidney stones, and joint inflammation (Sivarajan \& Balachandran 1994). In our preliminary study, methanol extract of $R$. cordifolia roots showed higher mosquitocidal activity than hexane and chloroform extracts against $C$. quinquefasciatus and $A$. aegypti. We report here the isolation and identification of an active molecule from root extracts of $R$. cordifolia against larvae and pupae of $C$. quinquefasciatus and $A$. aegypti.

\section{Material and Methods}

\section{Insect rearing}

Culex quinquefasciatus and Aedes aegypti larvae were reared in tap water at $27 \pm 2^{\circ} \mathrm{C}, 75-85 \% \mathrm{RH}$ with13:11 L/D photoperiod. Larvae were fed with dog biscuits and Brewer's yeast in the ratio of 3:2. Pupae were transferred from the rearing trays to plastic cups $(250 \mathrm{~mL})$ containing tap water and placed in breeding cages $(60 \times 60 \times 60 \mathrm{~cm}$ dimension) for adult emergence. Adults were fed with wet raisins and $10 \%$

Table 1 Lethal concentrations (in ppm) of different fractions of Rubia cordifolia methanol extract against larvae of Culex quinquefasciatus.

\begin{tabular}{|c|c|c|c|c|c|c|c|c|c|c|}
\hline \multirow[t]{2}{*}{ Mosquito species } & \multirow[t]{2}{*}{ Treatment } & \multirow[t]{2}{*}{$\mathrm{LC}_{50}(\mathrm{ppm})$} & \multicolumn{2}{|c|}{ 95\% confidence limit } & \multirow[t]{2}{*}{$\mathrm{LC}_{90}(\mathrm{ppm})$} & \multicolumn{2}{|c|}{$95 \%$ confidence limit } & \multirow[t]{2}{*}{ Intercept \pm SE } & \multirow[t]{2}{*}{ Slope \pm SE } & \multirow[t]{2}{*}{$\chi^{2}$} \\
\hline & & & $\mathrm{LL}$ & UL & & LL & UL & & & \\
\hline \multirow[t]{23}{*}{ Culex quinquefasciatus } & Fraction 1 & 5.60 & 5.0 & 6.25 & 16.05 & 12.99 & 22.05 & $2.9 \pm 0.25$ & $2.8 \pm 0.31$ & $3.3^{*}$ \\
\hline & Fraction 2 & 3.53 & 3.16 & 3.87 & 7.26 & 6.52 & 8.32 & $2.7 \pm 0.25$ & $4.0 \pm 0.3$ & $5.6^{*}$ \\
\hline & Fraction 3 & 4.32 & 3.83 & 4.80 & 11.27 & 9.62 & 14.08 & $3.03 \pm 0.24$ & $3.08 \pm 0.31$ & $2.6^{*}$ \\
\hline & Fraction 4 & 17.72 & 11.84 & 50.72 & 167.27 & 55.85 & 3673.75 & $3.35 \pm 0.25$ & $1.31 \pm 0.32$ & $1.3^{*}$ \\
\hline & Fraction 5 & 15.86 & 11.48 & 32.22 & 99.02 & 43.33 & 702.53 & $3.06 \pm 0.27$ & $1.61 \pm 0.33$ & $2.3^{*}$ \\
\hline & Fraction 6 & 8.15 & 6.78 & 10.80 & 47.90 & 27.06 & 154.82 & $3.48 \pm 0.23$ & $1.66 \pm 0.30$ & $2.0^{*}$ \\
\hline & Fraction 7 & 11.93 & 9.12 & 20.72 & 87.18 & 39.15 & 576.83 & $3.40 \pm 0.24$ & $1.48 \pm 0.31$ & $2.3^{*}$ \\
\hline & Fraction 8 & 7.06 & 6.19 & 8.26 & 25.53 & 18.35 & 44.56 & $3.05 \pm 0.25$ & $2.29 \pm 0.31$ & $5.9^{*}$ \\
\hline & Fraction 9 & 10.38 & 8.46 & 14.77 & 53.19 & 29.84 & 171.42 & $3.16 \pm 0.25$ & $1.80 \pm 0.31$ & $4.1^{*}$ \\
\hline & Fraction 10 & 8.93 & 7.50 & 11.68 & 42.30 & 25.70 & 110.09 & $3.19 \pm 0.25$ & $1.89 \pm 0.31$ & $4.6^{*}$ \\
\hline & Fraction 11 & 11.28 & 8.88 & 17.76 & 69.70 & 34.77 & 320.82 & $3.29 \pm 0.25$ & $1.62 \pm 0.31$ & $1.2^{*}$ \\
\hline & Fracton 12 & 8.84 & 7.24 & 12.30 & 55.54 & 29.69 & 210.78 & $3.47 \pm 0.24$ & $1.60 \pm 0.30$ & $4.4^{*}$ \\
\hline & Fraction 13 & 9.92 & 8.24 & 13.39 & 45.08 & 27.07 & 120.45 & $3.05 \pm 0.26$ & $1.94 \pm 0.32$ & $1.6^{*}$ \\
\hline & Fraction 14 & 10.02 & 8.29 & 13.70 & 47.05 & 27.77 & 131.72 & $3.08 \pm 0.26$ & $1.90 \pm 0.32$ & $0.9^{*}$ \\
\hline & Fraction 15 & 10.93 & 8.79 & 16.11 & 57.87 & 31.45 & 204.14 & $3.16 \pm 0.25$ & $1.77 \pm 0.32$ & $0.5^{*}$ \\
\hline & Fraction 16 & 6.97 & 6.04 & 8.30 & 28.77 & 19.70 & 56.10 & $3.24 \pm 0.24$ & $2.08 \pm 0.30$ & $2.8^{*}$ \\
\hline & Fraction 17 & 12.58 & 10.01 & 19.07 & 56.48 & 31.60 & 182.89 & $2.83 \pm 0.28$ & $1.96 \pm 0.34$ & $1.6^{*}$ \\
\hline & Fraction 18 & 10.91 & 8.89 & 15.55 & 51.53 & 29.54 & 155.69 & $3.02 \pm 0.26$ & $1.90 \pm 0.32$ & $0.3^{*}$ \\
\hline & Fraction 19 & 3.59 & 3.19 & 3.96 & 7.93 & 7.05 & 9.24 & $2.92 \pm 0.24$ & $3.72 \pm 3.72$ & $5.5^{*}$ \\
\hline & Fraction 20 & 7.17 & 6.22 & 8.56 & 29.01 & 19.90 & 56.26 & $3.19 \pm 0.24$ & $2.11 \pm 0.30$ & $5.9^{*}$ \\
\hline & Fraction 21 & 4.00 & 3.50 & 4.48 & 10.91 & 9.28 & 13.74 & $3.22 \pm 0.23$ & $2.94 \pm 0.31$ & $0.2^{*}$ \\
\hline & Fraction 22 & 10.87 & 8.60 & 16.83 & 68.14 & 34.17 & 309.60 & $3.33 \pm 0.25$ & $1.60 \pm 0.31$ & $0.2^{*}$ \\
\hline & Fraction 23 & 5.11 & 4.41 & 5.84 & 19.14 & 14.42 & 30.50 & $3.41 \pm 0.23$ & $2.23 \pm 0.29$ & $2.6^{*}$ \\
\hline
\end{tabular}

$\mathrm{LC}_{50}$ - lethal concentration that kills $50 \%$ of the exposed larvae, $\mathrm{LC}_{90}$ - lethal concentration that kills $90 \%$ of the exposed larvae.

LL lower limit (95\% confidence limit), UL upper limit (95\% confidence limit).

${ }^{*} p \leq 0.05$, level of significance of chi-square values. 
Table 2 Lethal concentrations (in ppm) of different fractions of Rubia cordifolia methanol extract against larvae of Aedes aegypti.

\begin{tabular}{|c|c|c|c|c|c|c|c|c|c|c|}
\hline \multirow[t]{2}{*}{ Mosquito species } & \multirow[t]{2}{*}{ Treatment } & \multirow[t]{2}{*}{$\mathrm{LC}_{50}(\mathrm{ppm})$} & \multicolumn{2}{|c|}{$95 \%$ confidence limit } & \multirow[t]{2}{*}{$\mathrm{LC}_{90}(\mathrm{ppm})$} & \multicolumn{2}{|c|}{$95 \%$ confidence limit } & \multirow[t]{2}{*}{ Intercept \pm SE } & \multirow[t]{2}{*}{ Slope \pm SE } & \multirow[t]{2}{*}{$\chi^{2}$} \\
\hline & & & $\mathrm{LL}$ & UL & & LL & UL & & & \\
\hline \multirow[t]{23}{*}{ Aedes aegypti } & Fraction 1 & 12.65 & 9.66 & 21.83 & 81.85 & 38.34 & 461.44 & $3.25 \pm 0.25$ & $1.58 \pm 0.31$ & $0.2^{*}$ \\
\hline & Fraction 2 & 3.86 & 3.47 & 4.23 & 8.28 & 7.38 & 9.61 & $2.72 \pm 0.25$ & $3.86 \pm 0.35$ & $0.7^{*}$ \\
\hline & Fraction 3 & 9.34 & 7.90 & 12.06 & 38.81 & 24.65 & 89.87 & $2.98 \pm 0.26$ & $2.07 \pm 0.32$ & $0.5^{*}$ \\
\hline & Fraction 4 & 9.28 & 7.87 & 11.94 & 38.05 & 24.35 & 86.48 & $2.97 \pm 0.26$ & $2.09 \pm 0.32$ & $3.09 *$ \\
\hline & Fraction 5 & 7.88 & 6.83 & 9.56 & 30.75 & 20.94 & 60.43 & $3.05 \pm 0.25$ & $2.16 \pm 0.31$ & $1.9^{*}$ \\
\hline & Fraction 6 & 7.86 & 6.96 & 9.15 & 24.07 & 17.97 & 38.73 & $2.63 \pm 0.27$ & $2.63 \pm 0.33$ & $2.8^{*}$ \\
\hline & Fraction 7 & 10.99 & 9.07 & 15.19 & 45.62 & 27.63 & 119.06 & $2.84 \pm 0.27$ & $2.07 \pm 0.33$ & $0.3^{*}$ \\
\hline & Fraction 8 & 12.01 & 9.39 & 19.28 & 69.92 & 35.17 & 311.54 & $3.1 \pm 0.2$ & $1.6 \pm 0.3$ & $0.7^{*}$ \\
\hline & Fraction 9 & 12.75 & 10.06 & 19.80 & 60.29 & 32.79 & 211.49 & $2.90 \pm 0.28$ & $1.89 \pm 0.34$ & $0.5^{*}$ \\
\hline & Fraction 10 & 13.40 & 10.21 & 23.17 & 78.30 & 37.81 & 396.38 & $3.11 \pm 0.26$ & $1.67 \pm 0.32$ & $0.3^{*}$ \\
\hline & Fraction 11 & 13.51 & 10.58 & 21.45 & 60.94 & 33.16 & 214.02 & $2.78 \pm 0.29$ & $1.95 \pm 0.35$ & $1.3^{*}$ \\
\hline & Fracton 12 & 12.89 & 10.07 & 20.63 & 65.40 & 34.29 & 255.48 & $2.98 \pm 0.27$ & $1.81 \pm 0.33$ & $0.05^{*}$ \\
\hline & Fraction 13 & 8.60 & 7.42 & 10.63 & 32.68 & 22.01 & 65.72 & $2.93 \pm 0.26$ & $2.21 \pm 0.32$ & $2.3^{*}$ \\
\hline & Fraction 14 & 7.74 & 6.85 & 9.05 & 24.57 & 18.17 & 40.29 & $2.72 \pm 0.26$ & $2.55 \pm 0.33$ & $4.9^{*}$ \\
\hline & Fraction 15 & 10.40 & 8.57 & 14.36 & 47.49 & 28.06 & 132.36 & $3.02 \pm 0.26$ & $1.94 \pm 0.32$ & $0.1^{*}$ \\
\hline & Fraction 16 & 11.28 & 9.24 & 15.87 & 47.81 & 28.46 & 130.56 & $2.84 \pm 0.27$ & $2.04 \pm 0.34$ & $0.5^{*}$ \\
\hline & Fraction 17 & 11.75 & 9.49 & 17.14 & 52.64 & 30.17 & 159.12 & $2.89 \pm 0.27$ & $1.96 \pm 0.33$ & $0.2^{*}$ \\
\hline & Fraction 18 & 7.77 & 6.81 & 9.20 & 26.88 & 19.24 & 47.23 & $2.88 \pm 0.26$ & $2.37 \pm 0.32$ & $1.7^{*}$ \\
\hline & Fraction 19 & 5.56 & 2.41 & 11.07 & 15.71 & 8.99 & 3613.04 & $2.88 \pm 0.43$ & $2.84 \pm 0.55$ & $6.0^{*}$ \\
\hline & Fraction 20 & 7.13 & 6.31 & 8.25 & 23.42 & 17.40 & 38.04 & $2.88 \pm 0.25$ & $2.48 \pm 0.32$ & $2.4^{*}$ \\
\hline & Fraction 21 & 5.67 & 5.10 & 6.30 & 15.47 & 12.69 & 20.75 & $2.78 \pm 0.25$ & $2.94 \pm 0.32$ & $3.8^{*}$ \\
\hline & Fraction 22 & 9.84 & 8.30 & 12.85 & 39.17 & 24.97 & 89.87 & $2.87 \pm 0.27$ & $2.13 \pm 0.33$ & $1.6^{*}$ \\
\hline & Fraction 23 & 6.30 & 5.65 & 7.07 & 18.0 & 14.34 & 25.51 & $2.75 \pm 0.25$ & $2.81 \pm 0.32$ & $3.4^{*}$ \\
\hline
\end{tabular}

${ }^{*} p \leq 0.05$, level of significance of chi-square values.

sucrose solution soaked in cotton. Adult females were deprived of sucrose for $6 \mathrm{~h}$ and then provided with a mouse placed in a breeding cage overnight for blood feeding. The ovitrap, containing water at the bottom and filter paper on the sides of a 500-mL plastic container was placed in the breeding cage, and the eggs were collected after 3 days. Third instars and pupae were used for the experiment.

\section{Plant material}

Roots of R. cordifolia (Fig 1) were collected from Kalakkad Mundanthurai Tiger Reserve forest (KMTR) in Southern Western Ghats of Tirunelveli District, India. The plant material was authenticated by Dr. S. Mutheeswaran, Taxonomist at Entomology Research Institute, Loyola College, Chennai. A voucher specimen (ERI-LA-MOS-304) was deposited in the herbarium of the institute.

\section{Extraction}

The roots were shade dried and coarsely powdered using an electric blender. The powdered root $(1 \mathrm{~kg})$ was extracted twice with methanol by cold percolation ( $48 \mathrm{~h}$ ). The extract was filtered through Whatman No. 1 filter paper and concentrated in a rotary evaporator and finally dried under vacuum.

\section{Chromatographic separation}

The methanol extract ( $91 \mathrm{~g}$ ) was subjected to column chromatography on a silica gel (100-200 mesh) column packed in hexane. The column was eluted with solvents of increasing polarity in the order hexane, ethyl acetate and methanol and their mixtures. Similar fractions were combined based on their TLC profiles. Finally, 23 fractions were obtained. Each fraction was subjected to mosquitocidal activity at the concentrations of 2.5, 5.0, 7.5 and $10 \mathrm{ppm}$. Fraction 2 eluted with hexane:ethyl acetate (90:10) showed significant mosquitocidal activity. Based on the bioassay results, fraction 2 was selected for further identification of the bioactive compound.

\section{Bioassays}

Larvicidal and pupicidal activities were evaluated using the method prescribed by World Health Organization (2005) 
Table 3 Lethal concentrations (in ppm) of different fractions of Rubia cordifolia methanol extract against pupae of Culex quinquefasciatus.

\begin{tabular}{|c|c|c|c|c|c|c|c|c|c|c|}
\hline \multirow[t]{2}{*}{ Mosquito species } & \multirow[t]{2}{*}{ Treatment } & \multirow[t]{2}{*}{$\mathrm{LC}_{50}(\mathrm{ppm})$} & \multicolumn{2}{|c|}{$95 \%$ confidence limit } & \multirow[t]{2}{*}{$\mathrm{LC}_{90}(\mathrm{ppm})$} & \multicolumn{2}{|c|}{ 95\% confidence limit } & \multirow[t]{2}{*}{ Intercept \pm SE } & \multirow[t]{2}{*}{ Slope \pm SE } & \multirow[t]{2}{*}{$\chi^{2}$} \\
\hline & & & $\mathrm{LL}$ & UL & & $\mathrm{LL}$ & UL & & & \\
\hline \multirow[t]{23}{*}{ Culex quinquefasciatus } & Fraction 1 & 4.78 & 4.24 & 5.31 & 13.23 & 11.02 & 17.25 & $3.02 \pm 0.24$ & $2.89 \pm 0.31$ & $4.9^{*}$ \\
\hline & Fraction 2 & 3.76 & 3.40 & 4.10 & 7.50 & 6.76 & 8.56 & $2.53 \pm 0.26$ & $4.27 \pm 0.37$ & $5.7^{*}$ \\
\hline & Fraction 3 & 11.85 & 9.03 & 20.84 & 90.59 & 39.82 & 651.11 & $3.44 \pm 0.24$ & $1.45 \pm 0.30$ & $0.9^{*}$ \\
\hline & Fraction 4 & 11.76 & 9.23 & 18.61 & 68.33 & 34.65 & 297.01 & $3.20 \pm 0.25$ & $1.67 \pm 0.32$ & $0.5^{*}$ \\
\hline & Fraction 5 & 8.66 & 7.39 & 10.92 & 36.15 & 23.39 & 80.14 & $3.06 \pm 0.25$ & $2.06 \pm 0.31$ & $3.1^{*}$ \\
\hline & Fraction 6 & 8.86 & 7.98 & 10.16 & 21.13 & 16.68 & 30.73 & $1.78 \pm 0.35$ & $3.39 \pm 0.41$ & $3.0^{*}$ \\
\hline & Fraction 7 & 9.28 & 7.87 & 11.94 & 38.05 & 24.35 & 86.48 & $2.97 \pm 0.26$ & $2.09 \pm 0.32$ & $3.0^{*}$ \\
\hline & Fraction 8 & 11.59 & 9.42 & 16.62 & 50.18 & 29.34 & 143.86 & $2.85 \pm 0.27$ & $2.01 \pm 0.34$ & $1.0^{*}$ \\
\hline & Fraction 9 & 10.08 & 8.41 & 13.51 & 43.10 & 26.46 & 108.62 & $2.96 \pm 0.26$ & $2.03 \pm 0.32$ & $0.6^{*}$ \\
\hline & Fraction 10 & 8.57 & 7.35 & 10.65 & 33.96 & 22.51 & 71.02 & $3.0 \pm 0.25$ & $2.14 \pm 0.32$ & $0.8^{*}$ \\
\hline & Fraction 11 & 10.66 & 8.92 & 14.24 & 40.80 & 25.82 & 95.40 & $2.73 \pm 0.28$ & $2.19 \pm 0.34$ & $1.6^{*}$ \\
\hline & Fracton 12 & 8.05 & 6.71 & 10.58 & 46.52 & 26.58 & 145.56 & $3.47 \pm 0.23$ & $1.68 \pm 0.30$ & $1.8^{*}$ \\
\hline & Fraction 13 & 8.14 & 7.06 & 9.91 & 30.93 & 21.12 & 60.33 & $2.98 \pm 0.25$ & $2.21 \pm 0.32$ & $3.8^{*}$ \\
\hline & Fraction 14 & 8.23 & 7.32 & 9.58 & 23.72 & 17.91 & 37.34 & $2.44 \pm 0.29$ & $2.78 \pm 0.35$ & $3.5^{*}$ \\
\hline & Fraction15 & 8.00 & 6.84 & 9.96 & 35.41 & 22.81 & 79.63 & $3.20 \pm 0.24$ & $1.98 \pm 0.31$ & $4.5^{*}$ \\
\hline & Fraction 16 & 11.79 & 9.48 & 17.40 & 54.61 & 30.80 & 172.59 & $2.93 \pm 0.27$ & $1.92 \pm 0.33$ & $1.2^{*}$ \\
\hline & Fraction 17 & 6.89 & 5.99 & 8.16 & 27.65 & 19.20 & 52.24 & $3.21 \pm 0.24$ & $2.12 \pm 0.30$ & $2.5^{*}$ \\
\hline & Fraction 18 & 6.12 & 5.28 & 7.19 & 26.59 & 18.35 & 51.30 & $3.41 \pm 0.23$ & $2.01 \pm 0.29$ & $1.5^{*}$ \\
\hline & Fraction 19 & 4.64 & 3.40 & 5.82 & 47.32 & 23.94 & 259.31 & $4.15 \pm 0.21$ & $1.27 \pm 0.28$ & $2.8^{*}$ \\
\hline & Fraction 20 & 7.69 & 6.62 & 9.39 & 32.50 & 21.55 & 68.15 & $3.18 \pm 0.24$ & $2.04 \pm 0.31$ & $2.3^{*}$ \\
\hline & Fraction 21 & 6.46 & 5.65 & 7.52 & 24.65 & 17.69 & 43.34 & $3.21 \pm 0.24$ & $2.20 \pm 0.30$ & $1.1^{*}$ \\
\hline & Fraction 22 & 6.28 & 5.47 & 7.32 & 24.87 & 17.69 & 44.60 & $3.28 \pm 0.24$ & $2.14 \pm 0.30$ & $3.0^{*}$ \\
\hline & Fraction 23 & 6.81 & 5.98 & 7.92 & 24.45 & 17.75 & 41.79 & $3.07 \pm 0.24$ & $2.30 \pm 0.31$ & $5.1^{*}$ \\
\hline
\end{tabular}

$\mathrm{LC}_{50}$ - lethal concentration that kills $50 \%$ of the exposed pupae, $\mathrm{LC}_{90}$ - lethal concentration that kills $90 \%$ of the exposed pupae.

LL lower limit ( $95 \%$ confidence limit), UL upper limit (95\% confidence limit).

${ }^{*} p \leq 0.05$, level of significance of chi-square values.

with slight modifications. Fractions were tested at 2.5, 5.0, 7.5 and $10 \mathrm{ppm}$ using acetone. Each treatment, including control, was replicated five times. Twenty third instars and pupae (In WHO protocol: 25 third instars used) of $C$. quinquefasciatus and $A$. aegypti were used for each replicate. Azadirachtin and temephos were used as positive controls, and acetone was used as a negative control. The dead larvae and pupae were registered after 24 -h exposure period. The percent mortality was calculated and subjected to corrections according to Abbott (1925) using:

$1-\frac{n \text { in T after treatment }}{n \text { in Cafter treatment }} \times 100$

where $n$ is the number of larvae, $T$ is the treated and $C$ is the control. The corrected percentage mortality value for each concentration was considered to estimate $\mathrm{LC}_{50}$ and $\mathrm{LC}_{90}$ values using US EPA probit analysis software (version 1.5).

\section{Identification of the active compound}

The active fraction 2 was crystallised from hexaneether mixture to get the active compound. The structure of the compound was elucidated on the basis of spectroscopic data. UV-vis spectrum was collected on a Shimadzu UV-Vis spectrophotometer in methanol. IR spectrum was obtained on a Perkin-Elmer FT-IR grating spectrophotometer in $\mathrm{KBr}$ disc. ${ }^{1} \mathrm{H}$ and ${ }^{13} \mathrm{C}$ NMR were produced on a Bruker Instrument at 400 and $100 \mathrm{MHz}$ in DMSO d6, respectively.

\section{Larvicidal and pupicidal activity of the compound}

The larvicidal and pupicidal activities of the isolated compound were performed as earlier mentioned. Test concentrations were $0.5,1.0,1.5$ and 2.0 ppm. 
Table 4 Lethal concentrations (in ppm) of different fractions of Rubia cordifolia methanol extract against pupae of Aedes aegypti.

\begin{tabular}{|c|c|c|c|c|c|c|c|c|c|c|}
\hline \multirow[t]{2}{*}{ Mosquito species } & \multirow[t]{2}{*}{ Treatment } & \multirow[t]{2}{*}{$\mathrm{LC}_{50}(\mathrm{ppm})$} & \multicolumn{2}{|c|}{$95 \%$ confidence limit } & \multirow[t]{2}{*}{$\mathrm{LC}_{90}(\mathrm{ppm})$} & \multicolumn{2}{|c|}{$95 \%$ confidence limit } & \multirow[t]{2}{*}{ Intercept \pm SE } & \multirow[t]{2}{*}{ Slope \pm SE } & \multirow[t]{2}{*}{$\chi^{2}$} \\
\hline & & & $\mathrm{LL}$ & UL & & $\mathrm{LL}$ & UL & & & \\
\hline \multirow[t]{23}{*}{ Aedes aegypti } & Fraction 1 & 10.16 & 8.56 & 13.33 & 39.18 & 25.07 & 89.02 & $2.79 \pm 0.27$ & $2.18 \pm 0.33$ & $0.7^{*}$ \\
\hline & Fraction 2 & 3.92 & 3.54 & 4.28 & 8.05 & 7.23 & 9.25 & $2.56 \pm 0.25$ & $4.09 \pm 0.36$ & $2.8^{*}$ \\
\hline & Fraction 3 & 7.32 & 6.58 & 8.30 & 20.19 & 15.84 & 29.42 & $2.48 \pm 0.28$ & $2.90 \pm 0.34$ & $4.9^{*}$ \\
\hline & Fraction 4 & 12.67 & 9.98 & 19.80 & 61.81 & 33.21 & 224.52 & $2.94 \pm 0.27$ & $1.86 \pm 0.33$ & $0.2^{*}$ \\
\hline & Fraction 5 & 14.69 & 10.99 & 26.72 & 82.15 & 39.15 & 433.97 & $2.99 \pm 0.27$ & $1.71 \pm 0.34$ & $0.2^{*}$ \\
\hline & Fraction 6 & 10.78 & 8.83 & 15.13 & 49.20 & 28.75 & 141.07 & $2.99 \pm 0.26$ & $1.94 \pm 0.32$ & $0.7^{*}$ \\
\hline & Fraction 7 & 9.42 & 7.96 & 12.21 & 39.25 & 24.84 & 91.69 & $2.98 \pm 0.26$ & $2.06 \pm 0.32$ & $1.4^{*}$ \\
\hline & Fraction 8 & 10.27 & 8.59 & 13.71 & 41.87 & 26.09 & 101.60 & $2.87 \pm 0.27$ & $2.10 \pm 0.33$ & $0.9^{*}$ \\
\hline & Fraction 9 & 10.16 & 8.44 & 13.72 & 44.40 & 26.94 & 115.43 & $2.98 \pm 0.26$ & $2.00 \pm 0.32$ & $0.6^{*}$ \\
\hline & Fraction 10 & 8.84 & 7.57 & 11.11 & 35.13 & 23.08 & 75.01 & $2.97 \pm 0.26$ & $2.13 \pm 0.32$ & $1.6^{*}$ \\
\hline & Fraction 11 & 8.04 & 7.09 & 9.48 & 25.73 & 18.81 & 43.20 & $2.70 \pm 0.27$ & $2.53 \pm 0.33$ & $1.9^{*}$ \\
\hline & Fracton 12 & 9.47 & 8.00 & 12.27 & 39.06 & 24.79 & 90.57 & $2.96 \pm 0.26$ & $2.08 \pm 0.32$ & $1.6^{*}$ \\
\hline & Fraction 13 & 9.73 & 8.32 & 12.32 & 34.51 & 23.13 & 70.31 & $2.69 \pm 0.28$ & $2.33 \pm 0.34$ & $1.2^{*}$ \\
\hline & Fraction 14 & 6.90 & 6.17 & 7.83 & 20.31 & 15.77 & 30.19 & $2.70 \pm 0.26$ & $2.73 \pm 0.32$ & $2.7^{*}$ \\
\hline & Fraction 15 & 9.50 & 8.15 & 11.94 & 33.722 & 22.74 & 67.65 & $2.72 \pm 0.28$ & $2.33 \pm 0.34$ & $0.9^{*}$ \\
\hline & Fraction 16 & 7.30 & 6.47 & 8.44 & 23.27 & 17.39 & 37.34 & $2.80 \pm 0.26$ & $2.54 \pm 0.32$ & $2.3^{*}$ \\
\hline & Fraction 17 & 11.64 & 9.29 & 17.52 & 58.97 & 32.05 & 207.26 & $3.06 \pm 0.26$ & $1.81 \pm 0.32$ & $0.3^{*}$ \\
\hline & Fraction 18 & 7.05 & 6.29 & 8.06 & 21.31 & 16.34 & 32.49 & $2.73 \pm 0.26$ & $2.66 \pm 0.32$ & $2.7^{*}$ \\
\hline & Fraction 19 & 4.90 & 4.35 & 5.44 & 13.58 & 11.28 & 17.80 & $3.00 \pm 0.24$ & $2.89 \pm 0.31$ & $4.5^{*}$ \\
\hline & Fraction 20 & 5.40 & 4.83 & 6.00 & 14.91 & 12.26 & 19.91 & $2.87 \pm 0.25$ & $2.90 \pm 0.31$ & $5.1^{*}$ \\
\hline & Fraction 21 & 5.46 & 4.88 & 6.08 & 15.30 & 12.51 & 20.63 & $2.88 \pm 0.25$ & $2.86 \pm 0.31$ & $5.8^{*}$ \\
\hline & Fraction 22 & 4.93 & 1.16 & 9.15 & 12.73 & 7.61 & 7189.93 & $2.84 \pm 0.49$ & $3.11 \pm 0.63$ & $7.8^{*}$ \\
\hline & Fraction 23 & 6.81 & 6.12 & 7.66 & 18.92 & 15.01 & 27.01 & $2.59 \pm 0.27$ & $2.88 \pm 2.88$ & $5.47^{*}$ \\
\hline
\end{tabular}

${ }^{*} p \leq 0.05$, level of significance of chi-square values.

\section{Results}

Bioassay results of crude chromatographic fractions

Among the 23 fractions screened, fraction 2 was found to be the most effective, with $\mathrm{LC}_{50}$ and $\mathrm{LC}_{90}$ values of $3.53,7.26 \mathrm{ppm}$ and $3.86,8.28 \mathrm{ppm}$ for larvae of $C$. quinquefasciatus and $A$. aegypti, respectively. Bioactivity of this fraction was followed by fraction 19 , which yielded $\mathrm{LC}_{50}$ and $\mathrm{LC}_{90}$ values of 3.59 , $7.93 \mathrm{ppm}$ and 5.56, $15.71 \mathrm{ppm}$ for larvae of C. quinquefasciatus and $A$. aegypti, respectively (Tables 1 and 2). The $\mathrm{LC}_{50}$ and $\mathrm{LC}_{90}$ values of fraction 2 for pupicidal activity were $3.76,7.50 \mathrm{ppm}$ and 3.92, $8.05 \mathrm{ppm}$ for pupae of C. quinquefasciatus and $A$. aegypti, respectively (Tables 3 and 4). All other fractions tested showed only moderate activity against the larvae and pupae of both mosquito species.
Fig 1 Rubia cordifolia whole plant (a) and its root (b).
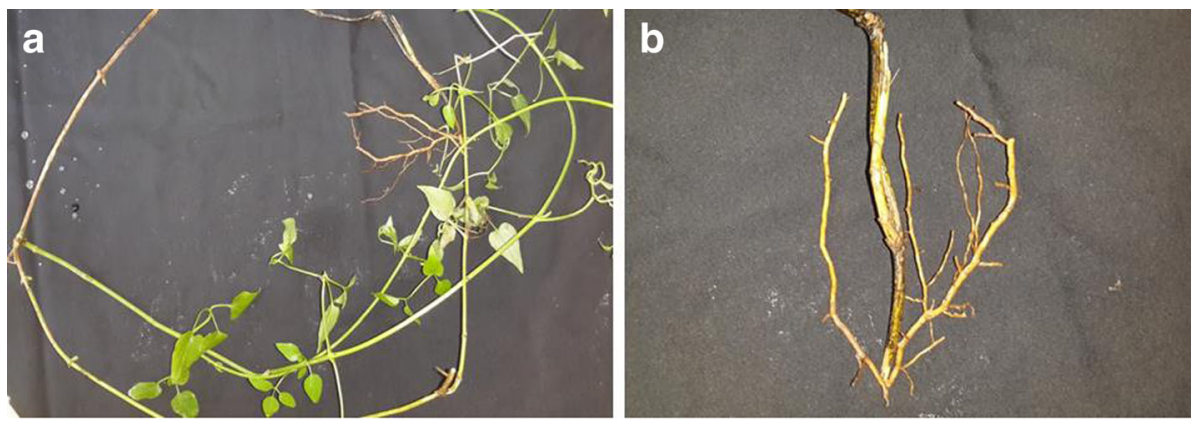


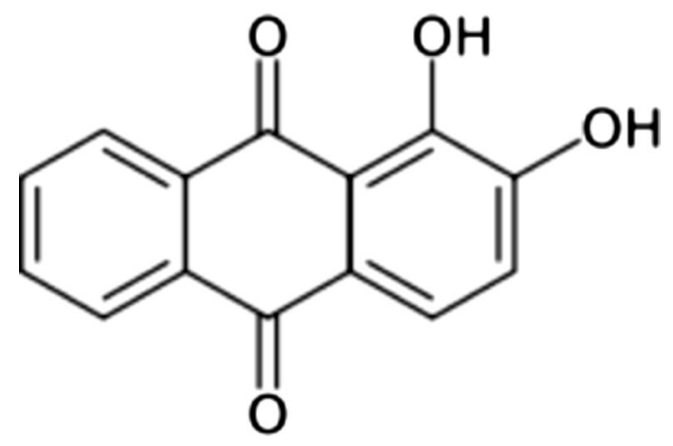

Fig 2 Structure of alizarin.

\section{Identification of the active compound}

The active molecule present in fraction 2 was identified as alizarin (1) [1,2-dihydroxyanthraquinone-9,10 anthraquinone] (Fig 2). It was obtained as reddish orange crystals from hexane-ether mixture: $\mathrm{mp} 148^{\circ} \mathrm{C}$. UV: $\lambda_{\max } \mathrm{MeOH} 249,270$, $435 \mathrm{~nm}$ (Fig 3). IR: $v_{\max } \mathrm{MeOH} \mathrm{KBr} 3422,3369$ (hydroxyl) 3074 (aromatic) 1662, 1631 (quinine carbonyl) 1588, 1457 (aromatic) 1340, 1294, 1191, 1037, 1017, 895, 840, 756, 711 (aromatic) (Fig 4). ${ }^{1} \mathrm{H}$ NMR ( $\delta$ DMSO d6, $400 \mathrm{MHz}$ ): 7.23 $(1 \mathrm{H}, \mathrm{d}, \mathrm{J}=8.4 \mathrm{~Hz}, \mathrm{H} 3), 7.66\left({ }^{1} \mathrm{H}, \mathrm{d}, \mathrm{J}=8.4 \mathrm{~Hz}, \mathrm{H}-4\right), 7.93(2 \mathrm{H}, \mathrm{m}, \mathrm{H} 6$ and $\mathrm{H} 7), 8.16\left({ }^{1} \mathrm{H}, \mathrm{m}, \mathrm{H} 8\right), 8.20(1 \mathrm{H}, \mathrm{m}, \mathrm{H} 5)(\mathrm{Fig} 5) .{ }^{13} \mathrm{C} \mathrm{NMR}(\delta$ DMSO d6, $100 \mathrm{MHz}$ ): 150.17 (C-1), 152.17 (C-2), 120.23 (C-3 and $\mathrm{C}_{4}$ ), 123.18 (C4a), 125.89 ( $\mathrm{C}_{5}$ and $\mathrm{C} 8$ ), 132.96 (C-8a), 134.52 (C-6) 133.46 (C-7), 188.19 (C-9) 115.65 (C-9a) 179.96 (C-10), 132.24 (C-10a) (Fig 6). The physical and spectroscopic data were comparable with those reported in the literature
(Ahmed et al 2014, Mahendra et al 2014). (See supplementary Material for Figs 3 to 6 ).

\section{Larval and pupal mortality caused by alizarin}

Exposure of larvae and pupae of $C$. quinquefasciatus and A. aegypti to alizarin increased mortality in a concentrationdependent manner. $\mathrm{LC}_{50}$ and $\mathrm{LC}_{90}$ values of alizarin for C. quinquefasciatus and $A$. aegypti larvae were 0.81 , $3.56 \mathrm{ppm}$ and 1.31, $6.04 \mathrm{ppm}$, respectively (Tables 5 and 6). Similarly, $L C_{50}$ and $L C_{90}$ values of alizarin for C. quinquefasciatus and $A$. aegypti pupae were 1.97, $4.79 \mathrm{ppm}$ and 2.05, $5.59 \mathrm{ppm}$, respectively (Tables 7 and 8). There was no mortality in controls, and all larvae and pupae were active and exhibited normal movement. Convulsions were observed at $2 \mathrm{ppm}$ of alizarin and dead larvae and pupae settled down as already reported (Reegan et al 2013).

\section{Discussion}

Mosquitoes are one of the most dangerous insects since they vector several pathogens to humans. Culex quinquefasciatus and $A$. aegypti are well established in tropical and subtropical regions, and they have also developed resistance to chemical insecticides (Tikar et al 2009, Llinás et al 2010, Mulyatno et al 2012, Chen et al 2013, Grisales et al 2013). Hence, plant extracts and isolated compounds would be good alternatives to control vector mosquitoes.

Table 5 Lethal concentrations of alizarin (in ppm) against larvae of Culex quinquefasciatus.

\begin{tabular}{|c|c|c|c|c|c|c|c|c|c|c|}
\hline \multirow[t]{2}{*}{ Mosquito species } & \multirow[t]{2}{*}{ Treatment } & \multirow[t]{2}{*}{$\mathrm{LC}_{50}(\mathrm{ppm})$} & \multicolumn{2}{|c|}{$95 \%$ confidence limit } & \multirow[t]{2}{*}{$\mathrm{LC}_{90}(\mathrm{ppm})$} & \multicolumn{2}{|c|}{$95 \%$ confidence limit } & \multirow[t]{2}{*}{ Slope \pm SE } & \multirow[t]{2}{*}{ Intercept \pm SE } & \multirow[t]{2}{*}{$\chi^{2}$} \\
\hline & & & $\mathrm{LL}$ & UL & & LL & UL & & & \\
\hline \multirow[t]{3}{*}{ Culex quinquefasciatus } & Alizarin & 0.81 & 0.59 & 1.01 & 3.56 & 2.41 & 8.443 & $2.1 \pm 0.3$ & $5.1 \pm 0.1$ & $4.1^{*}$ \\
\hline & Azadirachtin & 0.28 & 0.12 & 0.37 & 0.55 & 0.46 & 0.66 & $4.3 \pm 1.1$ & $7.4 \pm 0.3$ & $0.1^{*}$ \\
\hline & Temephos & 0.65 & 0.56 & 0.73 & 1.62 & 1.42 & 1.93 & $3.2 \pm 0.3$ & $5.5 \pm 0.1$ & $1.7^{*}$ \\
\hline
\end{tabular}

${ }^{*} p \leq 0.05$, level of significance of chi-square values.

Table 6 Lethal concentrations of alizarin (in ppm) against larvae of Aedes aegypti.

\begin{tabular}{|c|c|c|c|c|c|c|c|c|c|c|}
\hline \multirow[t]{2}{*}{ Mosquito species } & \multirow[t]{2}{*}{ Treatment } & \multirow[t]{2}{*}{$\mathrm{LC}_{50}(\mathrm{ppm})$} & \multicolumn{2}{|c|}{ 95\% confidence limit } & \multirow[t]{2}{*}{$\mathrm{LC}_{90}(\mathrm{ppm})$} & \multicolumn{2}{|c|}{$95 \%$ confidence limit } & \multirow[t]{2}{*}{ Slope \pm SE } & \multirow[t]{2}{*}{ Intercept \pm SE } & \multirow[t]{2}{*}{$\chi^{2}$} \\
\hline & & & LL & UL & & LL & UL & & & \\
\hline \multirow[t]{3}{*}{ Aedes aegypti } & Alizarin & 1.31 & 1.05 & 1.72 & 6.04 & 3.5 & 21.86 & $3.4 \pm 0.4$ & $4.7 \pm 0.1$ & $3.3^{*}$ \\
\hline & Azadirachtin & 0.34 & 0.22 & 0.43 & 1.04 & 0.90 & 1.27 & $2.6 \pm 0.3$ & $6.2 \pm 0.1$ & $3.7^{*}$ \\
\hline & Temephos & 0.92 & 0.11 & 1.66 & 1.82 & 1.17 & 646.1 & $4.3 \pm 0.9$ & $5.1 \pm 0.1$ & $2.4^{*}$ \\
\hline
\end{tabular}

Positive control values-Reegan et al 2014

${ }^{*} p \leq 0.05$, level of significance of chi-square values. 
Table 7 Lethal concentrations of alizarin (in ppm) against pupae of Culex quinquefasciatus.

\begin{tabular}{|c|c|c|c|c|c|c|c|c|c|c|}
\hline \multirow[t]{2}{*}{ Mosquito species } & \multirow[t]{2}{*}{ Treatment } & \multirow[t]{2}{*}{$\mathrm{LC}_{50}(\mathrm{ppm})$} & \multicolumn{2}{|c|}{$95 \%$ confidence limit } & \multirow[t]{2}{*}{$\mathrm{LC}_{90}(\mathrm{ppm})$} & \multicolumn{2}{|c|}{$95 \%$ confidence limit } & \multirow[t]{2}{*}{ Slope \pm SE } & \multirow[t]{2}{*}{ Intercept \pm SE } & \multirow[t]{2}{*}{$\chi^{2}$} \\
\hline & & & LL & UL & & LL & UL & & & \\
\hline \multirow[t]{3}{*}{ Culex quinquefasciatus } & Alizarin & 1.97 & 1.74 & 2.21 & 4.79 & 4.14 & 5.79 & $2.4 \pm 0.2$ & $4.0 \pm 0.1$ & $5.4^{*}$ \\
\hline & Azadirachtin & 0.47 & 0.41 & 0.52 & 0.80 & 0.72 & 0.92 & $5.6 \pm 0.7$ & $6.8 \pm 0.2$ & $0.3^{*}$ \\
\hline & Temephos & 0.75 & 0.68 & 0.82 & 1.52 & 1.37 & 1.74 & $4.1 \pm 0.3$ & $5.5 \pm 0.1$ & $4.8^{*}$ \\
\hline
\end{tabular}

${ }^{*} p \leq 0.05$, level of significance of chi-square values.

Table 8 Lethal concentrations of alizarin (in ppm) against pupae of Aedes aegypti.

\begin{tabular}{|c|c|c|c|c|c|c|c|c|c|c|}
\hline \multirow[t]{2}{*}{ Mosquito species } & \multirow[t]{2}{*}{ Treatment } & \multirow[t]{2}{*}{$\mathrm{LC}_{50}(\mathrm{ppm})$} & \multicolumn{2}{|c|}{$95 \%$ confidence limit } & \multirow[t]{2}{*}{$\mathrm{LC}_{9 \circ}(\mathrm{ppm})$} & \multicolumn{2}{|c|}{$95 \%$ confidence limit } & \multirow[t]{2}{*}{ Slope \pm SE } & \multirow[t]{2}{*}{ Intercept \pm SE } & \multirow[t]{2}{*}{$\chi^{2}$} \\
\hline & & & LL & UL & & LL & UL & & & \\
\hline \multirow[t]{3}{*}{ Aedes aegypti } & Alizarin & 2.05 & 1.71 & 2.84 & 5.59 & 3.69 & 13.77 & $2.9 \pm 0.5$ & $4.1 \pm 0.1$ & $3.3^{*}$ \\
\hline & Azadirachtin & 0.58 & 0.51 & 0.65 & 1.25 & 1.12 & 1.44 & $3.8 \pm 0.3$ & $5.9 \pm 0.1$ & $3.6^{*}$ \\
\hline & Temephos & 1.00 & 0.21 & 1.89 & 2.21 & 1.38 & 962.19 & $3.7 \pm 0.7$ & $4.9 \pm 0.1$ & $4.1^{*}$ \\
\hline
\end{tabular}

Positive control values-Reegan et al 2014

${ }^{*} p \leq 0.05$, level of significance of chi-square values.

In the present study, fraction 2 from the methanol extract of roots of $R$. cordifolia eluted with hexane:ethyl acetate (90:10) recorded good mosquitocidal activity against A. aegypti followed by fraction 19. $\mathrm{LC}_{50}$ and $\mathrm{LC}_{90}$ values for fraction 2 for third instars of $C$. quinquefasciatus and A. aegypti corroborated earlier findings of Muthu et al (2012) of the potential of plant extracts-Clerodendrum phlomidis-as sources of active molecules with insecticide activity against $C$. quinquefasciatus and $A$. aegypti.

The present study revealed that alizarin belongs to the anthraquinone group. Alizarin exhibited good larvicidal and pupicidal activities against both mosquito species. The activity was higher against $C$. quinquefasciatus than in A. aegypti. The estimated $\mathrm{LC}_{50}$ values for larvae and pupae of both mosquitoes are close to those reported by Han et al (2013) for lansiumamide B against early fourth instars of A. albopictus.

In conclusion, the fraction 2 and the isolated compound alizarin from methanol extract of $R$. cordifolia roots produced good activity against larvae and pupae of $C$. quinquefasciatus and $A$. aegypti. These results suggested that the isolated compound alizarin could be used in mosquito control programmes.

Electronic supplementary material The online version of this article (doi:10.1007/s13744-016-0386-x) contains supplementary material, which is available to authorized users.

Acknowledgments The authors are thankful to Entomology Research Institute, Loyola College for financial assistance and facilities. This project was supported by King Saud University, Deanship of Research, Addiriyah Chair for Environmental Studies.

\section{References}

Abbott WS (1925) A method of computing the effectiveness of an insecticide. J Econ Entomol 18:265-267

Ahmed FA, Monem AE, Sharaf MA, Sofy MR, Elhaw MH (2014) Quantitative analysis and isolated main active material of Convolvulus althaeoides and Convolvulus stachydifolius var. villosus. Researcher 6:1-10

Akram DS, Ahmed S (2005) Dengue fever. Infect Dis J 14:124-125

Bayen S (2012) Occurrence, bioavailability and toxic effects of trace metals andorganic contaminants in mangrove ecosystems: a review. Environ Int 48:84-101

Chen CD, Nazni WA, Lee HL, Norma-Rashid Y, Lardizabal ML, SofianAzirun M (2013) Temephos resistance in field Aedes (Stegomyia) albopictus (Skuse) from Selangor Malaysia. Trop Biomed 30:220-230

Devi Priya M, Siril EA (2014) Traditional and modern use of Indian Madder Rubia cordifolia L. An overview. Int J Pharm Sci Rev Res 25: 154-164

Grisales N, Poupardin R, Gomez S, Fonseca-Gonzalez I, Ranson H, Lenhart A (2013) Temephos resistance in Aedes aegypti in Colombia compromises dengue vector control. PLoS Negl Trop Dis 7:1-10

Han Y, Li L, Hao W, Tang M, Wan S (2013) Larvicidal activity of lansiumamide $B$ from the seeds of Clausena lansium against Aedes albopictus (Diptera: Culicidae). Parasitol Res 112:511-516

Harrington LC, Scott TW, Lerdthusnee K, Coleman RC, Costero A, Clark GG, Jones JJ, Kitthawee S, Kittayapong P, Sithiprasasna R, Edman JD (2005) Dispersal of the dengue vector Aedes aegypti within and between rural communities. Am J Trop Med Hyg 72:209-220

Holder P (1999) The mosquitoes of New Zealand and their animal disease significance. Surveillance 26:12-15

Kannan R (2012) State Records Most Dengue Cases and Deaths this Year. The Hindu (National News Paper), http://www.thehindu. $\mathrm{com} /$ news/national/tamil-nadu/state-records-most-denguecases-and-deaths-this-year/article4003294.ece\#comments Accessed 14 Aug 2015 
Kannathasan K, Senthilkumar A, Venkatesalu V (2011) Mosquito larvicidal activity of methyl-p-hydroxybenzoate isolated from the leaves of Vitex trifolia Linn. Acta Trop 120:115-118

Llinás GA, Seccacini E, Gardenal CN, Licastro S (2010) Current resistance status to temephos in Aedes aegypti from different regions of Argentina. Mem Inst Oswaldo Cruz Rio de Janeiro 105:113-116

Madhu SK, Shaukath AK, Vijayan VA (2010) Efficacy of bioactive compounds from Curcuma aromatic against mosquito larvae. Acta Trop 113:7-11

Mahendra AA, Bandra GK, Chandanie R (2014) A study of some anthraquionens of Rubia cordifolia L. incorporated in to Pinda oil: an ayurvedic medicinal oil used for tropical application in dermatological and inflammatory conditions. Int J Res Ayurveda pharm 5:334338

Mulyatno KC, Yamanaka A, Ngadino Konishi E (2012) Resistance of Aedes aegypti L. larvae to temephos in Surabaya, Indonesia. Southeast Asian J Top Med Public Health 43:29-33

Muthu C, Reegan AD, Kingsley S, Ignacimuthu S (2012) Larvicidal activity of pectolinaringenin from Clerodendrum phlomidis L. against Culex quinquefasciatus Say and Aedes aegypti L. (Diptera: Culicidae). Parasitol Res 111:1059-1065

NICD (1990) Proceedings of the national seminar on operation research on vector control in filariasis. National Institute of Communicable Diseases, New Delhi

Reegan AD, Kinsalin AV, Paulraj MG, Ignacimuthu S (2013). Larvicidal, ovicidal and repellent activities of marine sponge Cliona celata (Grant) extracts against Culex quinquefasciatus Say and Aedes aegypti L. (Diptera: Culicidae). ISRN Entomol 1-8

Reegan AD, Gandhi MR, Paulraj MG, Balakrishna K, Ignacimuthu S (2014) Effect of niloticin, a protolimonoid isolated from Limonia acidissima $\mathrm{L}$.
(Rutaceae) on the immature stages of dengue vector Aedes aegypti L. (Diptera: Culicidae). Acta Trop 139:67-76

Reegan AD, Gandhi MR, Paulraj MG, Ignacimuthu S (2015) Ovicidal and oviposition deterrent activities of medicinal plan extracts against Aedes aegypti L. and Culex quinquefasciatus Say mosquitoes (Diptera: Culicidae). Osong Public Health Res Perspect 6:64-69

Shaalan EA, Canyon D, Younes MWF, Abdel-Wahab H, Mansour AH (2005) A review of botanical phytochemicals with mosquitocidal potential. Environ Int 31:1149-1166

Sivarajan VV, Balachandran I (1994) Ayurvedic drugs and their plant sources. Oxford and India Book House Publishing Co Pvt Ltd, New Delhi, p 496

Sutthanont N, Choochote W, Tuetun B, Junkum A, Jitpakdi A, Chaithong U, Riyong D, Pitasawat B (2010) Chemical composition and larvicidal activity of edible plant-derived essential oils against the pyrethroidsusceptible and resistant strains of Aedes aegypti (Diptera: Culicidae). J Vector Ecol 35:106-115

Tikar SN, Kumar A, Prasad GB, Prakash S (2009) Temephos-induced resistance in Aedes aegypti and its cross-resistance studies to certain insecticides from India. Parasitol Res 105:57-63

World Health Organization (1984) Lymphatic filariasis. WHO Technical Report Series. Geneva: WHO; p.702. http://apps.who.int/iris/ bitstream/10665/39063/1/WHO_TRS_702.pdf Accessed 14 Aug 2015

World Health Organization (2005) Guidelines for laboratory and field testing of mosquito larvicides. WHO Geneva, WHO/CDS/WHOPES/ GCDPP/2005.13 http://apps.who.int/iris/bitstream/10665/69101/1/ WHO_CDS_WHOPES_GCDPP_2005.13.pdf Accessed 14 Aug 2015

Yakob L, Walker T (2016) Zika virus outbreak in the Americas: the need for novel mosquito control methods. Lancet Glob Health S2214$109 X(16) 00048-6$ 